\title{
Le jeu de voix dans des textes sur le travail enseignant : des pistes pour comprendre la morphogenèse de l'agir du professeur
}

\author{
Eliane Gouvêa Lousada \\ Université de São Paulo - USP \\ elianelousada@uol.com.br
}

\section{Introduction : la polyphonie et la morphogenèse de l'agir}

Cet article a pour but de présenter une analyse discursive des textes trouvés dans une situation de travail enseignant, de manière à contribuer pour une meilleure compréhension du travail du professeur de langues étrangères, à travers l'étude des pratiques langagières et de leur influence dans l'agir du professeur.

Le cadre théorique et méthodologique qui sous-tend cette étude est celui de l'interactionnisme sociodiscursif (Bronckart, 1997, 2004, 2006), dont un des objectifs est d'étudier la problématique de l'agir humain à partir des pratiques langagières. La vision de langue adoptée dans cette étude se base sur les concepts bakhtiniens (Bakhtine, 1977, 1997) de dialogisme et de polyphonie, selon lesquels l'altérité est constitutive du langage, celui-ci est donc toujours traversé par « l'autre ». On s'est également basé sur un autre cadre théorique, celui des Sciences du Travail, plus spécifiquement, l'Ergonomie de l'Activité (Amigues, 2002, 2003, 2004; Saujat, 2001, 2002, 2004) et la Clinique de l'Activité (Clot, 1999, 2001; Clot et al., 2001; Faïta, 2002, 2004, 2005). Finalement, en ce qui concerne l'analyse discursive des textes, on montrera l'analyse de la polyphonie dans le discours du professeur en question. Pour ce faire, les études de Bakhtine/Voloshinov (1977) et Bakhtine (1997) ont été consultées, ainsi que d'autres recherches qui proposent des procédures pour identifier les différentes voix dans les discours (AuthierRevuz, 2001 ; Maingueneau, 1991, 1996, 2001).

Pour atteindre l'objectif de cette étude, on a analysé la situation de travail enseignant d'un professeur de français comme langue étrangère qui enseignait à des étudiants de niveau avancé dans une école de français de São Paulo, SP, Brésil. Le corpus choisi est celui des auto-confrontations ${ }^{1}$, c'est-à-dire, quatre discussions filmées entre un professeur-formateur et un professeur à propos du visionnage de quatre cours donnés par le professeur. La transcription de ces discussions a été l'objet d'une analyse discursive des voix qui traversent le discours du professeur et qui pourraient avoir une influence sur la morphogenèse de son agir. Elles fonctionneraient comme un «texte pré-existant» qui prescrit le travail du professeur, à côté d'autres instances prescriptives, telles que les programmes du cours, les normes de l'école, les textes officiels, entre autres.

\section{Cadre théorique}

Parmi les principes de l'interactionnisme socio-discursif, il convient de rappeler que, selon Bronckart (2004), les textes peuvent contribuer au processus de reconfiguration de l'agir humain, vu qu'ils reconstituent le cadre social dans lequel les actions se développent. C'est la raison pour laquelle ils contribuent à la morphogénèse de l'ensemble des préconstruits collectifs ou des faits sociaux. Cependant, l'agir humain n'est pas accessible par la simple observation des conduites humaines observables (Bronckart, 2004). Il ne peut être appréhendé qu'à travers les interprétations, surtout verbales, fournies par les actants. Autrement dit, l'agir n'existe que dans les processus interprétatifs, d'où la nécessité de l'analyser à partir des textes qui le commentent. Voilà pourquoi on peut dire que, pour la compréhension du travail en tant que forme d'agir et pour comprendre la morphogenèse de l'agir, il faut que nous ayons accès aux textes qui présentent les interprétations des actants sur cet agir. 
Il semble également nécessaire de mentionner que la ligne théorique adoptée considère que, selon Bakhtine/Voloshinov (1977), l'énoncé/le texte est toujours traversé par des voix, dont le degré d'explicitation peut varier. On part ainsi du principe selon lequel le phénomène de la polyphonie a lieu dans n'importe quel texte, même dans ceux d'apparence plus monologique. Pour détecter des voix moins explicites $^{2}$, des auteurs comme Authier-Revuz (2001) et Maingueneau (2001) ont été consultés.

Le discours indirect libre peut révéler des voix plus implicites et donc plus difficiles à identifier étant donné qu'il est seulement visible à travers un désaccord entre deux voix qui sont complètement mélangées, de sorte que l'identification des énonciateurs est seulement perceptible à travers une bonne compréhension du contexte de production du texte. Maingueneau $(1991,1996)$ et Authier-Revuz $(2001)$ mentionnent encore le présupposé comme un élément capable de dévoiler l'existence d'une voix autre que celle qui peut être facilement identifiée. De même, Maingueneau (1996) cite les propositions et/ou conceptions évidentes comme une manière d'identifier la polyphonie et de mieux comprendre l'hétérogénéité constitutive du texte.

Finalement, la modalisation autonymique cherche à identifier les non-coïncidences du dire (AuthierRevuz, 2001:20; Maingueneau, 2001:159), qui concernent, par exemple, les expressions qui indiquent une distance ou une nécessité d'accord entre les co-énonciateurs. Selon Authier-Revuz (2001:20), l'hétérogénéité constitutive est considérée comme une non-cö̈ncidence du dire, car les commentaires méta-énonciatifs ne sont pas inscrits dans la structure syntaxique, mais dans des images et des masques qui montrent «la négociation obligatoire de tout énonciateur avec le fait des non-cö̈ncidences fondamentales qui traversent son dire ». Cet auteur partage ces formes purement interprétatives de l'hétérogénéité constitutive en quatre items, parmi lesquels nous en décrivons une (Authier-Revuz, 2001:21) : la non-coïncidence du discours avec lui-même. Celle-ci est considérée comme constitutive à partir des notions d'interdiscours et de dialogisme de Bakhtine, vu que tout mot est habité par le discours de l'autre, car il a été produit dans le déjà dit des autres discours. Plusieurs catégories existent, parmi lesquelles : extérieur approprié à l'objet du dire, dans laquelle sont utilisés des mots d'un autre lieu, d'une autre époque, d'une autre théorie, d'une autre personne, etc ; type d'autre, dans laquelle sont employés des mots d'une autre langue, région, époque, registre, "sociolecte" ${ }^{3}$, discours théorique, position politique, etc. Selon Authier-Revuz (2001:193), il s'agit de cas de discours qui ont une apparence monologique, en niant l'extériorité discursive.

En ce qui concerne les Sciences du Travail, notamment l'Ergonomie de l'Activité et la Clinique de l'Activité, il semble important de présenter deux concepts traditionnels dans ce domaine: travail prescrit et travail réalisé. Le travail prescrit concerne ce qui est demandé par l'institution, l'école. Le travail réalisé pourrait être considéré comme celui qui est effectivement réalisé par les travailleurs, c'est-à-dire la partie du travail qui peut être « vue ». Finalement, on aurait un autre concept proposé dans le cadre de la Clinique de l'Activité (Clot, 2001), celui de réel de l'activité. L'activité réelle comprendrait tout ce qui englobe le travail, non seulement le travail qui est effectivement réalisé, mais aussi ce qu'on aurait voulu faire, toutes les possibilités qui ont été empêchées de se réaliser. Dans cette perspective, le travail enseignant serait bien plus complexe que ce que l'on voit dans la salle de classe, qui semble être effectivement réalisé par le professeur. Il comprendrait, selon Saujat (2002), l'organisation du travail prescrite par l'institution scolaire (programmes, manuels, outils didactiques, etc.); le travail de reconception fait par les collectifs de travail ; le retravail, ou les auto-prescriptions, par chaque enseignant pour adapter les prescriptions à sa salle de classe.

C'est de cette manière que l'on peut comprendre les contributions individuelles de chaque enseignant au renouvellement du métier: en ré-inventant les manières de faire la classe, d'utiliser les artefacts disponibles, l'enseignant introduit des contributions stylistiques. Comme le souligne Saujat,

Les contributions stylistiques de chaque enseignant, liées à la façon dont il s'approprie singulièrement un patrimoine commun, peuvent alors être reconnues comme des moyens de revitaliser le genre professionnel, qu'on définira ici comme un ensemble en mouvement de manières de faire, de dire et de penser le métier. (Saujat, 2002 : 73) 
Ainsi, le genre professionnel peut évoluer grâce aux contributions du style de chaque enseignant, si ces contributions reviennent au collectif de travail.

\section{Contexte de la recherche et analyse des données}

Cette recherche a été réalisée dans une école de français langue étrangère (FLE) de São Paulo, Brésil. Le professeur qui s'est porté volontaire pour cette étude enseignerait pour la première fois le cours avancé. Il a donné son accord pour avoir quatre de ses cours filmés, ce qui a constitué le matériel pour visionnage et discussions postérieures. Ces discussions ont été également filmées ${ }^{4}$ et constituent une partie des données analysées présentées dans cet article. Pour atteindre l'objectif plus large de la recherche, un objectif plus restreint a été formulé: - identifier les sources de l'agir du professeur, à travers l'identification des voix, plus ou moins explicites, qui interviennent dans son discours et qui montrent l'influence des différentes prescriptions dans son agir.

Pour identifier ces voix, on a cherché des indices qui peuvent être remarqués au travers de mots qui appartiennent au jargon professionnel, c'est-à-dire des mots appartenant au domaine de la didactique (Authier-Revuz, 2001), mais aussi d'autres indices tels que la non-cö̈ncidence du dire avec lui-même (Authier-Revuz, 2001), les négations, les présupposés (Authier-Revuz, 2001 ; Maingueneau, 1991, 1996) et les propositions et/ou conceptions évidentes (Maingueneau, 1996).

Ainsi, on verra par la suite que, à travers la voix du professeur, d'autres voix peuvent être entendues, telles que :

- La voix de l'école: qui exprime des convictions et des prescriptions qui ont leur origine à l'école ;

- La voix de la didactique (didactique des langues, du FLE) : qui véhicule des aspects basés sur ces théories ;

- La voix du collectif de travail plus restreint : la voix de l'ensemble de professeurs de l'école ;

- La voix du métier: la voix du collectif de travail plus large, basée sur la tradition du métier de professeur, qui exprime la partie sous-entendue du travail du professeur et qui, en général, n'est pas explicite.

On a considéré comme voix de l'école les occasions où le professeur reproduit quelque chose qui coïncide avec le réseau de discours qui y sont produits. La voix de la didactique, en revanche, intervient quand le professeur aborde des concepts de la didactique du FLE, qu'il a appris pendant sa formation ou à l'école où la recherche a été réalisée. La voix du collectif de travail (plus restreint) apparaît dans des moments où le professeur parle au nom de l'ensemble des professeurs de l'école, en montrant la situation de travail dans laquelle ils vivent. Finalement, on a considéré comme la voix du métier, les moments où le professeur évoque des manières "d'être » et de "faire» qui semblent avoir leur origine dans sa conception de travail du professeur et qui ne viennent pas forcément de l'école ni des conceptions de la didactique. Ce sont souvent des conceptions qui ne sont pas explicitées dans les formations en didactique et qui peuvent être identifiées à travers des propositions évidentes, des présupposés. Or, les propositions évidentes, les vérités absolues, montrent des conceptions sur le travail du professeur qui sont acceptées par tous et révèlent donc les idées plus profondes qui sont à l'origine du métier. Dans ces cas-là, comme l'affirme Clot (1999), il s'agit du «métier » qui parle, en faisant voir les conceptions de fond qui soustendent son travail.

Dans l'exemple ci-dessous, nous pouvons voir l'interférence de la voix de l'école qui a créé le cours avancé (CA) et qui a établi ainsi un jargon propre à l'école (l'intégration des manuels) par rapport à ce cours. Le professeur évoque la difficulté représentée par le cours qui préconise l'intégration des livres didactiques et des modules.

\footnotetext{
4P19 - Et moi je me rends compte, je comprends beaucoup... mieux l'idée quoi... oui moi je serais disponible pour continuer parce que là le CA comme il est l'idée c'est l'intégration des manuels, de culture et de langue et ce découpage l'idée c'est la fusion entre ... et c'est ça justement que je
} 
voulais saisir et je pense maintenant que je comprends mieux...

Dans un autre moment, le professeur mentionne l'horaire où il arrivait à l'école en montrant qu'il suivait ce qui était préconisé par le directeur de l'unité où la recherche a été réalisée et qu'il avait incorporé la voix de l'école où il travaille. Le fait de dire qu'il n'arrivait jamais en même temps que les élèves montre, à travers la procédure de la négation, que d'autres professeurs pourraient le faire :

4P24 - moi je crois que moi j'étais un petit peu en avance ... j'arrivais à arriver 10 minutes avant quelque chose comme ça parce qu'il faut préparer tout... j'arrivais jamais en même temps qu'eux...

Le présupposé (Authier-Revuz, 2001; Maingueneau, 1991, 1996) selon lequel d'autres professeurs pourraient arriver en même temps que les élèves permet aussi d'identifier la voix que, par la connaissance du contexte de la recherche, on reconnaît comme étant celle du directeur de l'unité qui préconisait, pendant les réunions, que les professeurs arrivent avant l'horaire du cours.

Dans le prochain exemple, c'est le discours indirect qui permet de détecter la voix de l'école, à travers la catégorie de l'hétérogénéité montrée :

4P3 - Tout de suite avec un certain recul tout de suite je me rends compte quand même que moi j'aurais dû travailler davantage des des la mise au tableau quoi... mais ce qu'il y a c'est que j'ai travaillé quand même pas mal écrit avec eux ... tu vois des formes de textes rendus et des comptes rendus... Il me semblait avoir entendu qu'il fallait pas trop insister sur l'écrit alors et du coup là je ne me suis pas trop arrêté enfin... mais je constate avec un certaine recul que j'aurais dû quand même exploiter de plus près leur production faire plus de mise au tableau...

La voix du collectif de travail plus restreint peut être remarquée quand le professeur parle au nom de ses collègues:

1P23 - oui oui je pense c'est vrai que chacun travaille un peu à sa façon t'as prévu t'as des documents chacun travaille un peu à sa façon bon il y a des avantages et des inconvénients mais je pense que tout le monde dans l'ensemble se sent un peu avalé par le nombre de documents de matériel quoi et finalement comment gérer tout ça et c'est pas facile et je pense que le mieux c'est c'est de préparer une fiche de compréhension orale ...

Le professeur mentionne, dans cet exemple, le fait que les professeurs travaillent chacun à sa manière, que tous se perdent dans la quantité de matériel didactique et qu'il n'est pas facile d'organiser tout cela. A travers le discours indirect libre, le professeur semble avoir exprimé ses propres opinions et sentiments, peut-être à partir des observations de ses collègues de travail qui doivent partager les mêmes plaintes. En fait, c'est le contact avec le collectif de travail de l'école qui a permis au professeur de parler en connaissance de cause, comme un membre de ce collectif.

Dans le prochain exemple, le professeur évoque la question de l'expression orale, en mentionnant qu'il a parlé à la place des élèves et il dévoile ainsi la voix de la didactique. Cette voix était souvent assumée par l'école où la recherche a été réalisée, étant donné qu'elle offrait souvent des formations en didactique du FLE, où le thème de la nécessité de prise de parole par les apprenants était souvent évoqué. On remarque la non-coïncidence du dire avec lui-même, car le professeur montre qu'il sait qu'il ne doit pas prendre la parole des élèves, mais pense quand même que ce qu'il a fait était correct, étant donné que les élèves auraient appris quelque chose.

$1 \mathrm{P} 2$ - La prise de parole là vraiment j'ai pris la parole un peu à leur place là vraiment je pense que ... ils n'avaient pas la parole facile et pourtant je les connais ils ont vraiment une bonne compréhension orale et cette vidéo n'est pas difficile je pense qu'ils sont capables de comprendre mais ils n'arrivent pas à articuler les réponses ...

$1 C 3-$ et les points positifs?

1P4 - Je pense qu'ils ont appris quelque chose je pense enfin même si c'est moi qui ai pris la parole je pense que j'ai réussi à les faire raisonner ils ont réussi à comprendre quel est le rôle et le parcours 
du chef d'orchestre ...

Dans l'exemple ci-dessous, la voix du métier semble indiquer des conduites appartenant au genre professionnel du métier de professeur, qui semblent être inscrites dans la mémoire impersonnelle et collective de ce métier (Clot, 1999, 2001). Les thèmes abordés par cette voix ne sont pas retrouvés de manière explicite dans les théories de la didactique et quand ils sont abordés à l'école où la recheche a été réalisée, ils sont vus comme des présupposés, des vérités incontestables. C'est le cas de la gestion du temps, qui semble préoccuper souvent le professeur. Il explique qu'il n'écrit pas au tableau, car ce serait long et il perdrait du temps. Cette affirmation révèle la conception selon laquelle le professeur ne doit pas perdre du temps :

1 C14 - ou mettre au tableau si tu mets au tableau c'est un peu comme la fiche ...

1P15 - je n'écris pas de questions au tableau parce que c'est un peu trop long je crois que c'est une perte de temps ...

Dans ce même exemple, c'est encore la négation (je n'écris pas au tableau) qui révèle le fait que d'autres professeurs écrivent au tableau. Or, le fait d'écrire au tableau est considéré comme un geste professionnel qui fait partie du métier d'enseignant, et c'est souvent la raison pour laquelle il n'est pas souvent repris dans des formations didactiques.

Dans la même auto-confrontation, le professeur reprend la question de la lenteur et de la nécessité de gagner du temps :

$2 \mathrm{P} 5$ - je pense que là j'ai beaucoup parlé et j'ai trouvé quand même que là mon discours était assez pertinent... j'ai essayé d'être le plus clair possible..... je vois pas je répète j'avais conscience que c'était un peu trop lent j'ai profité pour gagner du temps il y avait des gens qui arrivaient ...

La voix du métier apparaît au travers de l'expression «j'avais conscience » qui, comme le verbe subjectif «avouer» (Maingueneau, 1996:99), montre que le professeur savait qu'un cours ne doit pas être trop lent. C'est encore le présupposé qui montre la nécessité de gagner du temps comme une vérité incontestable. Il convient de rappeler que la question de la gestion du temps n'était pas abordée à l'école où la recherche a été réalisée et elle n'était pas non plus abordée explicitement dans les formations didactiques.

Voilà pourquoi on assume que lorsqu'il s'agit d'un présupposé, d'une vérité présentée comme incontestable, il peut s'agir de la voix du métier. A travers cette voix, le profeseur montre qu'il doit prendre des décisions pendant le cours, face à des situations imprévues, guidé par des représentations qu'il a du métier et qu'il a construites au fil de son histoire, en tant qu'apprenant et aussi en tant qu'enseignant. On voit ainsi que le métier se constitue d'une partie sous-entendue, qui n'est pas explicite et qui est rarement abordée dans des programmes de formation, comme le soulignent l'Ergonomie de l'Activité (Saujat, 2002, 2004 ; Amigues, 2003, 2004) et la Clinique de l'Activité (Clot, 1999, 2001 ; Faïta, 2002, 2004).

\section{Résultats des analyses}

On peut conclure que les auto-confrontations réalisées ont quelques caractéristiques : 1'usage du pronom on, comme mentionné par Clot $\left(2005^{5}\right)$, mais aussi d'autres pronoms (tu, chacun) et d'autres unités lexicales (tout le monde) qui véhiculent la voix du collectif de travail de l'école où la recherche a été réalisée. Il peut s'agir également de la voix du métier, si l'on considère que les situations évoquées par cette voix font partie aussi des préoccupations du collectif plus large de travail, de tous les enseignants; la présence fréquente de plusieurs voix comme celles de la didactique, de la didactique du FLE, de l'école, du collectif restreint de travail, du métier de professeur. On peut également remarquer que le professeur répond aux prescriptions venues de l'école et de la didactique, soit en étant d'accord, soit en émettant son 
désaccord par rapport à ces voix. Le tableau (1) ci-dessous montre le nombre d'occurrences de voix dans le discours du professeur.

\begin{tabular}{|l|l|}
\hline Voix de l'école & 3 \\
\hline Voix du collectif & 2 \\
\hline Voix de la didactique & 7 \\
\hline Voz du métier & 11 \\
\hline
\end{tabular}

Tableau 1: Occurrence des voix.

On voit que les voix qui prédominent dans les paroles du professeur viennent de la Didactique et, surtout, du métier. Cependant, à côté de ces données quantitatives, il convient de faire une analyse qualitative et de se demander avec quelles voix le professeur est d'accord, avec lesquelles il ne l'est pas? Quelles prescriptions sont acceptées, lesquelles sont refutées ? C'est ce que montre le tableau (2) :

\begin{tabular}{|l|l|l|}
\hline Voix de la & $\begin{array}{l}\text { Montre qu'il connaitt la prescription de parler moins et de faire parler les élèves, } \\
\text { Daidactique n'est pas toujours d'accord avec cela et affirme l'importance du temps de } \\
\text { parole du professeur. }\end{array}$ \\
\hline $\begin{array}{l}\text { Voix } \quad \mathbf{d e} \\
\text { l'école }\end{array}$ & $\begin{array}{l}\text { Montre que l'enseignant est accord avec le programme du CA, avec la fusion entre } \\
\text { les deux manuels, mais le trouve difficile. Il est d'accord avec la prescription } \\
\text { d'arriver dix minutes avant le cours. }\end{array}$ \\
\hline $\begin{array}{ll}\text { Voix } \quad \mathbf{d u} \\
\text { collectif }\end{array}$ & $\begin{array}{l}\text { Parle au nom du collectif de travail, en soulignant le caractère individuel du travail } \\
\text { du professeur et la difficulté à gérer l'excès de matériel. }\end{array}$ \\
\hline $\begin{array}{l}\text { Voix } \quad \mathbf{d u} \\
\text { métier }\end{array}$ & $\begin{array}{l}\text { Exprime les convictions du professeur, ou les autopresciptions, avec lesquelles il } \\
\text { est souvent d'accord. Les thèmes sont liés à la nécessité de gagner du temps/ne pas } \\
\text { perdre de temps, profiter des moments, être dynamique, clair, etc. }\end{array}$ \\
\hline
\end{tabular}

Tableau 2: Le dialogue des voix.

Si l'on analyse le tableau ci-dessus, on peut remarquer qu'il y a deux voix que le professeur accepte, mais avec des restrictions : celle de la Didactique et celle de l'école. Par contre, il semble accepter sans restriction les voix du collectif et du métier. On peut voir également que les derniers items montrent que le travail enseignant englobe la conception, l'organisation et la régulation du milieu de travail des élèves, pour qu'il y ait apprentissage. Dans cette perspective, cette voix s'approche plus des aspects pris en compte par l'Ergonomie de l'Activité, c'est-à-dire, les manières de faire le travail, que des aspects plus cognitifs pris en compte, en général, par la Didactique.

Ainsi, contrairement à ce que l'on peut penser dans les programmes de formation, on voit que le professeur n'est pas simplement quelqu'un qui exécute simplement les prescriptions de la didactique et de l'école. Comme les analyses des discours le montrent, c'est le dialogue et la négociation avec les différentes voix, à travers les accords et les désaccords avec les prescriptions, qui se trouvent à l'origine de l'agir du professeur. Les différentes « couches » de prescriptions laissent des marques qui peuvent être appréhendées dans des textes produits par le professeur sur son agir, quand il verbalise son travail réel. C'est à travers la re-conception, la réélaboration des prescriptions que le professeur cherche à être efficace dans son travail. 


\section{Considérations finales: prendre en compte la voix du métier}

Selon Clot (1999:34) et Faïta (2005:62), le travail aurait une partie explicite et une autre partie, implicite, sous-entendue, qui d'habitude n'est pas énoncée. Cette partie sous-entendue est inscrite dans la tradition sociohistorique du métier et pré-organise les conduites des travailleurs/professeurs (Amigues, 2004; Clot, 1999). De cette façon, tout comme les genres de textes/discours (Bakhtine, 1997) ont des dimensions prescriptives qui conditionnent l'élaboration du texte, les genres professionnels ont également des dimensions prescriptives qui pré-organisent le travail, mais qui ne sont pas explicites, pour être économiques et donc efficaces.

Si l'on revient aux analyses, une des voix présentes dans les textes produits pendant les autoconfrontations est la voix du métier. On vu que les conceptions du professeur sont souvent liées au travail d'organisation du milieu et de régulation du travail des apprenants, et non à la conception et à l'élaboration de programmes et contenus. Tous ces aspects sont révélés par la voix du métier, qui conteste maintes fois la voix de l'école et de la didactique. Ainsi, cette voix met en évidence les «manières de faire » le travail du professeur et surtout celles qui ne sont pas déterminées par la didactique du FLE. Ainsi, il semble que ce soit justement cette voix qui véhicule la partie sous-entendue du travail du professeur, celle qui n'est pas énoncée et qui constitue, selon Clot (1999:34), l'âme sociale du travail, issue d'une véritable tradition du métier de professeur. Ainsi, l'identification de la voix du métier semble être un pas important pour l'explicitation du genre professionnel.

Une autre voix mise en évidence dans nos analyses est celle du collectif qui exprime la situation de travail vécue par les professeurs de l'école, rapportée par le professeur volontaire. Elle semble montrer le travail réel du professeur, stressé par l'excès de matériel et par le manque de temps pour gérer tout cela. Voilà pourquoi un plus grand dialogue avec les instances prescriptives semble essentiel pour équilibrer les désirs et la faisabilité de réalisation de ces désirs.

Le genre professionnel du métier de professeur, inscrit dans une véritable tradition de la profession, rend superflue l'explicitation de quelques prescriptions. Cependant, puisque ces manières d'agir ne sont pas une application stricte des théories de la didactique, ce serait intéressant de les expliciter dans la formation initiale. Même si elles sont disponibles dans le collectif de travail, leur explicitation pourrait faciliter l'insertion de nouveaux professeurs ou même contribuer à aider des professeurs en difficulté. Ces manières d'agir seraient un objet d'apprentissage pour l'appropriation du genre professionnel, de même que les manières de faire le texte sont un objet d'apprentissage pour l'appropriation des genres textuels.

D'autre part, le genre professionnel doit évoluer, ce qui se passe avec le contact avec les différents styles de chaque travailleur. Or, la voix du métier est celle qui véhicule le plus de désaccords par rapport à la didactique. Il faudrait donc discuter de ces désaccords avec la didactique, étant donné que c'est la prise de conscience sur les différentes manières faire qui peuvent faire évoluer le genre professionnel. Dans cette perspective, la discussion avec le collectif de travail sur les différentes manières de faire et sur la partie sous-entendue du travail du professeur est plus utile pour faire évoluer le genre que seulement l'exposition des théories de la didactique.

Voilà pourquoi il semble important d'accorder une place à cette voix du métier, à cette manière de faire le travail du professeur comme une manière de souder le collectif et comme une alternative à la solitude du métier de professeur. On donnerait ainsi les moyens pour que l'enseignant puisse en discuter et essayer de comprendre la partie sous-entendue du métier enseignant. Dans cette perspective, prendre en compte la voix du métier pourrait être une piste pour de nouvelles recherches dans le domaine du développement de professeurs.

\section{Références bibliographiques}

Amigues, R. (2002). L'enseignement comme travail. In: Bressoux, P. (ed.). Les stratégies d'enseignement en situation d'interaction. Note de synthèse pour Cognitique. Paris: Programme Ecole et Sciences Cognitives, 243262. 
Amigues, R. (2003). Pour une approche ergonomique de l'activité enseignante. In: Amigues, R; Faïta, D.; Kherroubi, M. (eds.). Métier enseignant, organisation du travail et analyse de l'activité. Skholê, hors série. 1, 5-16.

Amigues, R. (2004). Trabalho do professor e trabalho de ensino. In: Machado, A. R. (ed.) O ensino como trabalho: uma abordagem discursiva. Londrina : Eduel, 35-54.

Authier-Revuz, J. (2001). Palavras incertas : as não-coincidências do dizer. Campinas: Editora da Unicamp.

Bakhtine, M/Voloshinov, V. (1977). Marxisme et philosophie du langage. Paris : Minuit.

Bakhtin, M. (1997). Estética da criação verbal. São Paulo : Martins Fontes.

Bronckart, J-P. (1997). Activité langagière, textes et discours: pour un interactionnisme socio-discursif. Neuchâtel: Delachaux et Niestlé.

Bronckart, J-P. (2004). Agir et discours en situations de travail. Genève: Université de Genève, FPSE.

Bronckart, J-P. (2006). Atividade de linguagem, discurso e desenvolvimento humano. Campinas: Mercado de letras.

Clot, Y. (1999). La fonction psychologique du travail. Paris: Presses Universitaires de France.

Clot, Y. (2001). Editorial. Clinique de l'activité et pouvoir d'agir, 146, 7-16.

Clot, Y. et al. (2001). Entretiens en auto-confrontation croisée: une méthode en clinique de l'activité. Clinique de l'activité et pouvoir d'agir, 146, 17-25.

Cuq, J-P. (2003). Dictionnaire de didactique du Français. Paris: CLE International.

Faïta, D. (2002). Análise das práticas linguageiras e situações de trabalho: uma renovação metodológica imposta pelo objeto. In: Souza-e-Silva, M. C. P.; Faïta, D. (eds.). Linguagem e trabalho: construção de objetos de análise no Brasil e na França. São Paulo: Cortez.

Faïta, D. (2004). Gêneros de discurso, gêneros de atividade, análise da atividade do professor. In: Machado, A. R. (Ed.). O ensino como trabalho: uma abordagem discursiva. Londrina: Eduel, 55-80.

Faïta, D. (2005). Análise dialógica da atividade profissional. Rio de Janeiro: Imprinta Express.

Maingueneau, D. (1991). L'analyse du discours: introduction aux lectures de l'archive. Paris: Hachette.

Maingueneau, D. (1996). Les termes clés de l'analyse du discours. Paris : Seuil.

Maingueneau, D. (2001). Análise de textos de comunicação. Tradução de Cecília P. de Souza-e-Silva e Décio Rocha. São Paulo : Cortez Editora.

Saujat, F. (2001). Co-analyse de l'activité enseignante et développement de l'expérience: du travail de chacun au travail de tous et retour. Clinique de l'activité et pouvoir d'agir, 146, 87-98.

Saujat, F. (2002). Systèmes d'apprentissage: systèmes d'évaluation. 2002. Thèse (Doctorat) - Université de Provence, Provence.

Saujat, F. (2004). O trabalho do professor nas pesquisas em educação: um panorama. In: Machado, A. R. (ed.). $O$ ensino como trabalho: uma abordagem discursiva. Londrina: Eduel, 3-34.

${ }^{1}$ Cette procédure de filmage des discussions entre chercheur et professeur, à partir de l'observation des vidéos des cours filmés, a été développée par des chercheurs de la Clinique de l'Activité (Clot, 1999, 2001).

2 L'analyse des voix plus explicites a été l'objet d'un article qui est sous presse.

3 Variations du langage, registres de langue, qui peuvent comprendre des spécificités lexicales, phonétiques et morphosyntaxiques (Cuq, 2003:222).

4 Pour une explication plus complète de la technique des auto-confrontations, voir Clot et al. (2001).

5 Communication orale, PUC/SP/Brésil. 